\title{
Integral Control of Humanoid Balance
}

\author{
Benjamin Stephens \\ The Robotics Institute \\ Carnegie Mellon University \\ Pittsburgh, PA 15213, USA \\ bstephensecmu . edu \\ http://Www. cs. cmu. edu/ bstephe1
}

\begin{abstract}
This paper presents a balance controller that allows a humanoid to recover from large disturbances and still maintain an upright posture. Balance is achieved by integral control, which decouples the dynamics and produces smooth torque signals. Simulation shows the controller performs better than other simple balance controllers. Because the controller is inspired by human balance strategies, we compare human motion capture and force plate data to simulation. A model tracking controller is also presented, making it possible to control complex robots using this simple control.
\end{abstract}

\section{INTRODUCTION}

A fundamental control problem for humanoids is balancing, which is related to the control of unstable systems such as inverted pendulums. We believe that balance is achieved by a set of decoupled controls that regulate the center of pressure and simultaneously ensure that the humanoid balances upright. In human balance experiments, it has been observed that people use a mixture of strategies for dealing with these disturbances. The ankle strategy fixes all joints except the ankle, and balances like a single inverted pendulum. The hip strategy is characterized by a large bending at the hips, which results in a repositioning of the center of mass [1]. These two strategies are illustrated in Fig. 1.

In Section II, we present a controller that is inspired by these human balance strategies and accounts for the limits on the location of the center of pressure to ensure that the robot can stand with its feet flat on the ground and withstand large disturbances. We begin by ignoring the presence of the feet and pretend there are no constraints on the joint torques. The torques generated by the balance controller determine the ideal positioning of the center of pressure. This information is fed into an integral controller which maintains the constraints on the center of pressure and keeps the robot standing upright. Our controller is simulated on a double inverted pendulum, which is subjected to a large external disturbance force and results are presented in Section III. The performance is compared to other controllers and to human balance experiment data.

Since humans behave like double inverted pendulums, we want our humanoid robots to also behave as such. For this reason, we present a model tracking control algorithm in Section IV. The algorithm makes use of operational points to define a relationship between the simple model system (e.g. the double inverted pendulum) and the more complex

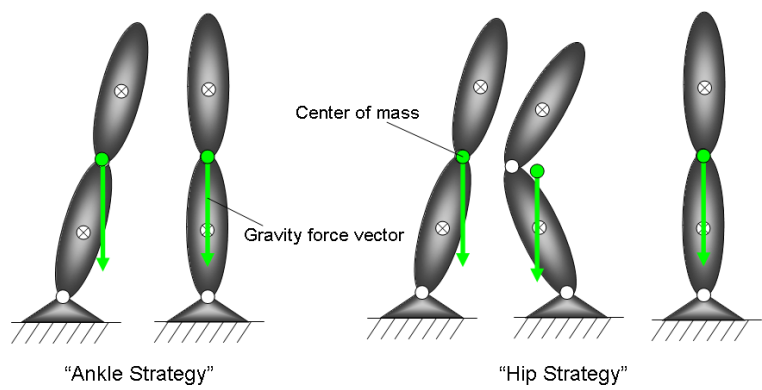

Fig. 1. The two strategies mimicked by our balance controller. The ankle strategy causes the robot to behave like a single inverted pendulum while the hip strategy allows the robot to use the gravitational force to help it balance

robot (e.g. a humanoid). We apply this algorithm to a 3-link planar biped robot in the sagittal plane and simulation results are presented in Section V.

\section{A. Related Work}

Humanoid robot researchers often describe balance and motion using simple models. Early work on balance and stability of dynamic bipeds was done by Vukobratovic, et. al. [2], followed by Golliday, et. al. [3] and Hemami, et. al. [4]. In these studies, the biped was usually represented by a planar double inverted pendulum with the base joint representing the stance foot and ankle joint.

The center of pressure $(\mathrm{CoP})$ is used as a measure of stability in bipeds. It represents the location of an equivalent force, equal to the integral of the pressure distribution under the foot, that is a measure of the tendency for the feet to rotate and come off the ground. The use of ground reference points, such as the CoP, has been present in almost every humanoid project. While the definition and usefulness of the $\mathrm{CoP}$ has been questioned [5] [6], it is still the dominant measure of stability used by many robots, including the highly successful Honda Asimo [7].

The constraints imposed on the ankle joint make humanoids behave like the acrobot [8], which consists of a series of inverted pendulums with all but the base joint actuated. By definition, the CoP of the acrobot is always fixed below the base joint, yet it can still balance itself. In humanoids, the location of the CoP is roughly proportional to the magnitude of the torque at the ankle. If the ankle torque 


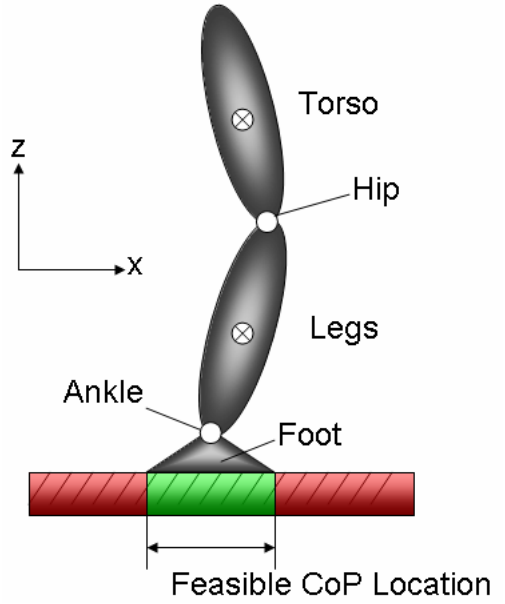

Fig. 2. A double inverted pendulum with foot can apply torques at the ankle and hip joints. The location of the center of pressure must be underneath the foot

is always commanded to be zero, then the CoP stays at zero as well, and the humanoid becomes an unstable acrobot.

Biomechanics researchers have subdivided human balance control into the hip strategy and the ankle strategy [9]. For the ankle strategy, all of the joints except for the ankles are fixed and balance is accomplished by torquing only the ankles. At some point, this becomes too difficult for the ankles and the hip strategy is employed. This is characterized by a large deflection at the hip and a quick centering of the center of mass over the ankle. Shifting the center of mass manipulates the moment created by the gravitational force, allowing the human to balance much like the acrobot.

Preview control [10], similar to model predictive control [11] and constrained optimal control [12], changes the inputs incrementally by simulating the system forward to prevent constraint violations. This kind of incremental control is necessary because the $\mathrm{CoP}$ imposes limits on the joint torques, namely the ankles, at each instant to keep the humanoid's feet flat on the ground. Integral control has previously been used to control humanoid balance [13] to decouple the dynamics of the center of pressure and the center of mass.

The idea of using intuitive controllers for simpler models on complex robots led to the development of operational space controls [14] and virtual model controls [15]. Both make use of the principle of virtual work and the Jacobian to perform actions in a task space. In operational space control, controllers are created to track trajectories of operational points and in virtual model control the joint torques create virtual forces at arbitrary points on the body. Khatib, et al. applied prioritized operational space controls to humanoids [16] to complete dynamic tasks while remaining in an upright posture. As first presented, the controller was better suited for arm motions or single support tasks, but was later organized into a general framework which included the necessary contact constraints for controlling humanoids [17].

\section{BALANCE CONTROL}

Balance in humanoids can be partially explained by the center of pressure, or CoP. This point must be away from the edges of the foot, or the foot will begin to rotate. Traditional control, such as linear quadratic regulators and PID controls do not account for this constraint, except by limiting the torque commands. In the presence of a large external disturbance force, these linear controllers would command more torque than the robot can deliver while keeping the foot flat on the ground. For this reason, we derive a controller that takes into account these constraints, ensuring that the robot remains balanced and upright with its feet flat on the ground.

The balance controller is derived below for fully-actuated, unconstrained planar dynamic systems with equations of motion of the form,

$$
M(\theta) \ddot{\theta}=\tau-N(\theta, \dot{\theta})
$$

where $\theta$ is a vector of joint angles, $M$ is the inertia matrix, $\tau$ are the joint torques, and $N$ is a vector containing the gravitational, centripetal and coriolis forces.

We use planar inverted pendulum models, like the one in Fig. 2, to present the controller. Even though there is no explicit foot in our model, it is assumed to be flat on the ground at all times, contributing no kinetic or potential energy. The definition of the center of pressure still applies as long as it remains within the area the foot would cover.

\section{A. Unconstrained Balance Control}

We initially ignore the presence of the foot, which imposes limits on the location of the CoP, and hence limits on the ankle torques. We use a two-part balance controller inspired by the hip and ankle strategies. The first controller is a simple full-state linear quadratic regulator (LQR). This optimal controller is found by linearizing the dynamics about vertical and it can be written as

$$
\tau_{\mathrm{LQR}}=-K_{\mathrm{LQR}}\left[\begin{array}{c}
\theta \\
\dot{\theta}
\end{array}\right]
$$

This controller always applies restoring torques to drive the joint angles to zero. This causes the robot to behave like a stiff inverted pendulum, similar to the ankle strategy. However, as the deflection at the ankle becomes larger, the center of mass moves outwards, creating a large moment due to gravity that the ankle torque must balance. For this reason, we add another controller to regulate the horizontal position of the center of mass over the ankle to minimize this moment, much like the hip strategy. The acceleration of the horizontal position of the center of mass, $x_{\mathrm{CM}}$, can be written as

$$
\ddot{x}_{\mathrm{CM}}=\dot{J}_{x} \dot{\theta}+J_{x} M^{-1}(\tau-N),
$$

where $J_{x}=\partial x_{\mathrm{CM}} / \partial \theta$. The regulator response we desire is $\ddot{x}_{\mathrm{CM}}=-k_{\mathrm{CMp}}\left(x_{\mathrm{CM}}-x_{\mathrm{CM}}^{d}\right)-k_{\mathrm{CMd}} \dot{x}_{\mathrm{CM}}$, which we can achieve by the following control,

$$
\begin{aligned}
\tau_{\mathrm{CM}}= & N-M J_{x}^{+}\left(\dot{J}_{x} \dot{\theta}+k_{\mathrm{CMp}}\left(x_{\mathrm{CM}}-x_{\mathrm{CM}}^{d}\right)\right. \\
& \left.+k_{\mathrm{CMd}} \dot{x}_{\mathrm{CM}}\right),
\end{aligned}
$$




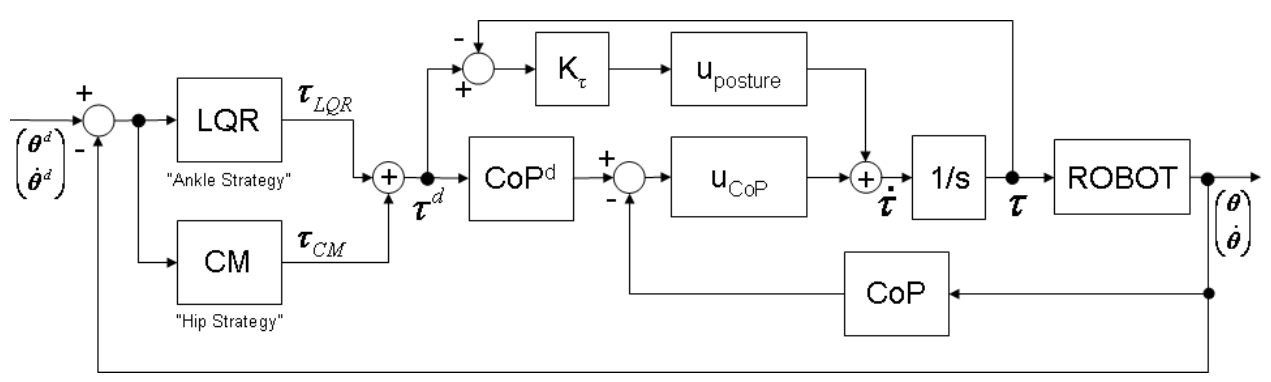

Fig. 3. Block diagram of balance controller

where $J_{x}^{+}$designates the pseudo-inverse of the matrix $J_{x}$ and $x_{\mathrm{CM}}^{d}$ is a desired center of mass location. For balance, the desired location should be over the ankle, so that the force of gravity points through the ankle joint and causes no moment. Unlike the LQR controller above, this controller will apply non-restoring torques. These non-restoring torques can result in a large deflection at the hip, characteristic of the hip strategy. In general, these controllers can be conflicting. However, the CM regulator only has an effect as the CM moves away from zero and cannot maintain posture alone, so both controllers are used simultaneously.

\section{B. CoP Regulator}

The location of the center of pressure is an important indicator of the robot's ability to balance because it represents the position of a single equivalent force on the foot. In order to keep the feet flat on the ground, this point has to be within the area under the foot. For our double inverted pendulum model of planar biped robots, the location of the center of pressure is given by an equation of the form,

$$
x_{\mathrm{CoP}}=\frac{\tau_{\text {ankle }}}{F_{\text {normal }}}=\frac{A \tau}{B+C \tau},
$$

where $A$ is a matrix that selects the ankle torque from the vector of torques, $\tau$. The normal force cancels the weight and downward acceleration of the center of mass, so $F_{\text {normal }}=$ $m_{\text {tot }}\left(\ddot{z}_{\mathrm{CM}}+g\right)$ and

$$
\begin{aligned}
& B=m_{\text {tot }}\left(\dot{J}_{z} \dot{\theta}-J_{z} M^{-1} N+g\right) \\
& C=m_{\text {tot }} J_{z} M^{-1}
\end{aligned}
$$

where $g=9.81 \mathrm{~m} / \mathrm{s}^{2}$ and $J_{z}$ is the Jacobian of the $z$ coordinate of the center of mass, and a function of joint angles only. The numerator of this equation is a function of only the torque at the ankle joint, meaning that if the ankle torque is zero then so is the CoP. This means that the limits on the location of the CoP approximately define the limits on the ankle torque. The problem with applying the unconstrained controllers above is that it may command an ankle torque that would violate these constraints. For this reason, we use the above controllers as a reference torque,

$$
\tau^{d}=\tau_{\mathrm{LQR}}+\tau_{\mathrm{CM}}
$$

We use this reference torque to compute an ideal center of pressure, $C o P^{*}$, using Eq.(5). If the required ankle torque is too large, this point will exceed the boundaries of the foot.
The actual desired center of pressure, $C o P^{d}$, is created by limiting this value to be under the foot.

To actually balance while tracking the desired center of pressure, we use a decoupled integral controller,

$$
u=u_{\mathrm{CoP}}+u_{\text {posture }}
$$

where $u_{\mathrm{CoP}}$ regulates the location of the CoP and $u_{\text {posture }}$ keeps the robot standing upright. The joint torques are the integral of this equation. The use of integral control creates smooth torque inputs and results in easily decoupled dynamics, as shown below.

We can track $x_{\mathrm{CoP}}^{d}$ using a feedback control that gives the response, $\dot{x}_{\mathrm{CoP}}=-k_{\mathrm{CoP}}\left(x_{\mathrm{CoP}}-x_{\mathrm{CoP}}^{d}\right)$. The dynamics of the $\mathrm{CoP}$ are found by taking a derivative of Eq.(5),

$$
\dot{x}_{\mathrm{CoP}}=D+E u
$$

where $u=\dot{\tau}$ is the new control input and

$$
\begin{aligned}
D & =-((\dot{B}+\dot{C} \tau) A \tau) / F^{2} \\
E & =(F A-C A \tau) / F^{2} \\
F & =B+C \tau
\end{aligned}
$$

According to Eq.(8), to achieve our desired response we must apply the control,

$$
u_{\mathrm{CoP}}=-E^{+}\left(D+k_{\mathrm{CoP}}\left(x_{\mathrm{CoP}}-x_{\mathrm{CoP}}^{d}\right)\right)
$$

The approximate effect of the pseudo-inverse is to command an ankle torque, since the center of pressure is largely determined by this torque. Using only Eq.(9), we can control the location of the CoP, but ankle control alone will not keep the robot standing upright. For posture control, we add extra controls that lie in $E^{*}=\operatorname{null}(E)$. Intuitively, this nullspace is essentially associated with all joints other than the ankle. According to Eq.(8), such controls will not affect the dynamics of the CoP. To control posture, we attempt to track the desired torque from Eq.(6), the unconstrained balance controller above. The resultant posture controller becomes

$$
u_{\text {posture }}=\sum_{i}\left(E_{i}^{* T} K_{\tau}\left(\tau^{d}-\tau\right)\right) E_{i}^{*}
$$

where $E_{i}^{*}$ is a unit basis vector of the null space and $K_{\tau}$ is a gain matrix that determines how closely the integrator tracks the reference torque, $\tau^{d}$. Because the null-space is associated with all joints except for the ankle, Eq.(10) does not change the ankle torque. However, the reference ankle 


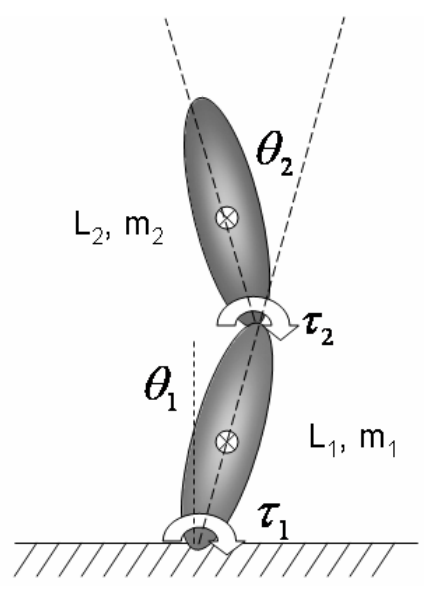

Fig. 4. Double inverted pendulum model used in this example. Parameters are $m_{1}=35 \mathrm{~kg}, m_{2}=35 \mathrm{~kg}, L_{1}=1 \mathrm{~m}$ and $L_{2}=1 \mathrm{~m}$
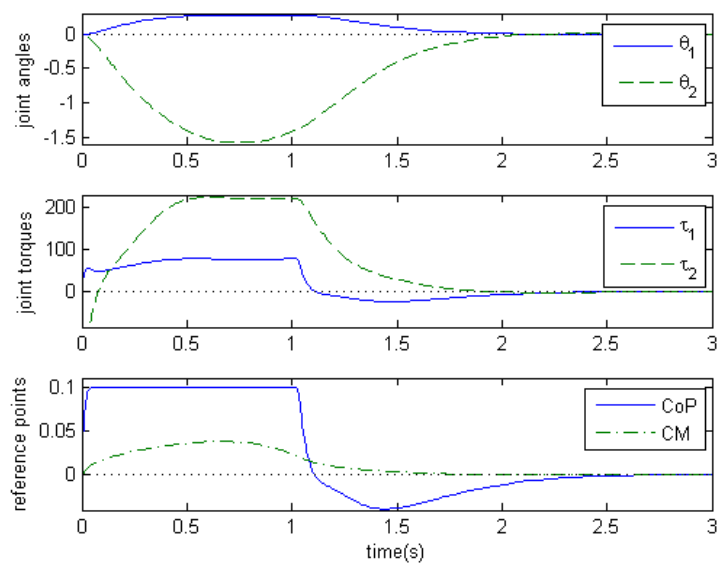

Fig. 5. Response to a $23 N s$ impulse push.

torque is not ignored, because it was used in the calculation of $C o P^{d}$, which is tracked using Eq.(9). The entire controller is summarized in the block diagram in Fig. 3.

\section{EXAmple: Double InVerted Pendulum}

This controller is applied to a planar double inverted pendulum robot, shown in Fig. 4. The center of mass of each link is at the center and there are joint torques at both the ankle and hip joints. The linear quadratic regulator controller was derived by writing linearized dynamics about the upright pose, $(\theta, \dot{\theta})^{T}=(0,0,0,0)^{T}$, as

$$
\left(\begin{array}{c}
\dot{\theta} \\
\ddot{\theta}
\end{array}\right)=A\left(\begin{array}{c}
\theta \\
\dot{\theta}
\end{array}\right)+B u
$$

For the properties of the model, given in Fig. 4, and the following manually-chosen $Q$ and $R$ matrices,

$$
Q=\operatorname{diag}\left[1 e^{4}, 1 e^{6}, 1,10\right] \quad \text { and } \quad R=\operatorname{diag}[100,100]
$$

the $K$ matrix became

$$
K_{\mathrm{LQR}}=\left[\begin{array}{cccc}
1383.0 & 347.6 & 508.2 & 151.0 \\
299.2 & 366.6 & 131.6 & 77.62
\end{array}\right]
$$

TABLE I

Control Parameters

\begin{tabular}{|l|r|}
\hline Parameter & Value \\
\hline$k_{\mathrm{CMp}}$ & 400 \\
$k_{\mathrm{CMd}}$ & 50 \\
$k_{\mathrm{CoP}}$ & 100 \\
$K_{\tau}$ & $\operatorname{diag}\left[1 e^{4}, 1 e^{4}\right]$ \\
\hline
\end{tabular}

Maximum Push Force Before Foot Rotation/Falling

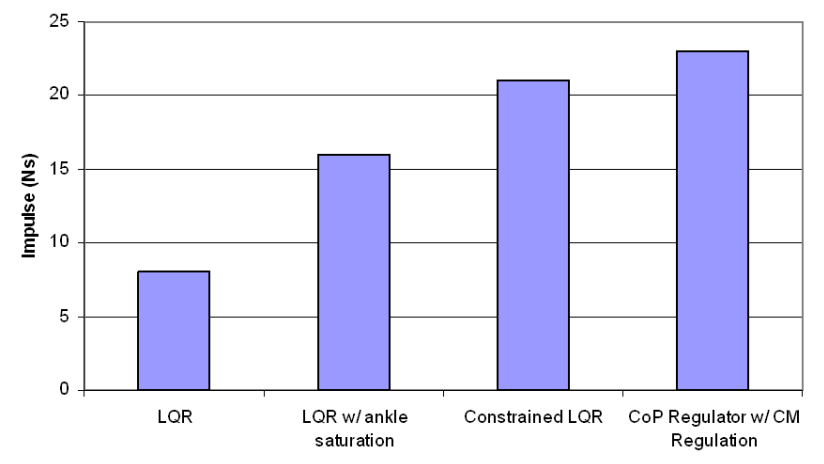

Fig. 6. Performance comparison of various controllers subject to a horizontal impulse.

The other parameters used for the controller are given in Table I.

Fig. 5 shows the response to an unknown disturbance, in this case an instantaneous $23 \mathrm{Ns}$ horizontal impulse to the center of the torso link. This impulse results in non-zero initial joint velocities. The CoP was constrained to be on the interval $x_{\mathrm{CoP}} \in[-0.1,0.1]$.

\section{A. Performance Comparison}

For comparison, we will use several other controllers that would commonly be used for this type of control problem and compare their performance to our controller. By performance, we mean the robot's ability to withstand large disturbance forces without breaking the constraints on the center of pressure and causing the foot to rotate or fall over. The three controllers we will compare our controller to are an unconstrained LQR controller, an LQR controller with a saturation limit on the ankle torque and a constrained LQR controller using receding horizon control. The same model and simulator were used for each controller. As before, horizontal pushes were applied to the center of mass of the upper link, and the magnitude was increased until the robot became unstable, either by the CoP moving to the edge of the foot or by falling over.

The results of this comparison are summarized in Fig. 6 . As expected, the unconstrained LQR controller performs the worst because it takes no care to avoid applying too large of an ankle torque and the CoP quickly moves to the edge of the foot. When a saturation limit on the ankle is imposed on the LQR controller, its performance is increased because it now accounts for CoP constraints, but cannot handle large 

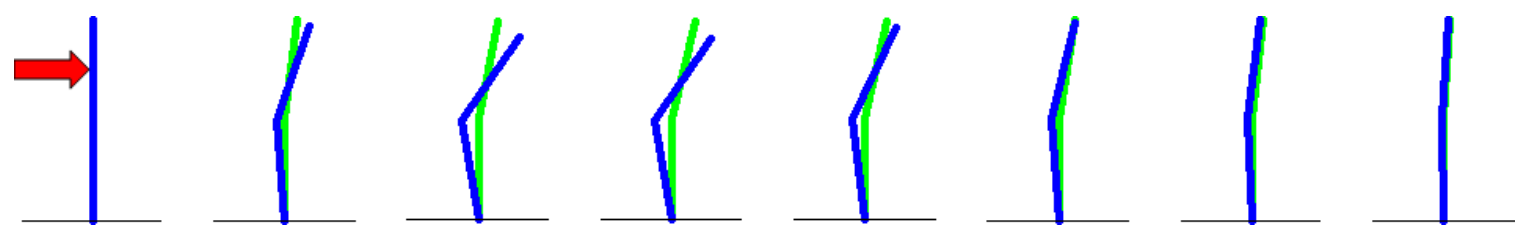

Fig. 7. Animation comparing the responses of the constrained LQR controller (light green) and our controller (dark blue) to a 17Ns impulse. Our controller produces a large bend at the hips, similar to the hip strategy used by humans

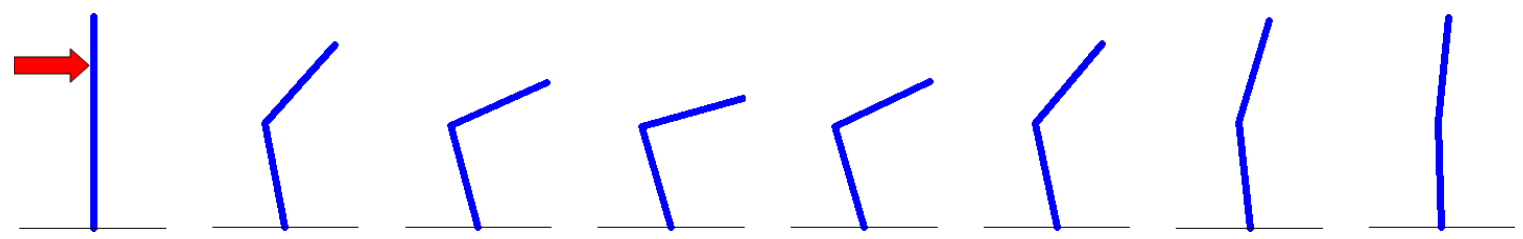

Fig. 8. Animation of the response to a $23 \mathrm{Ns}$ impulse.
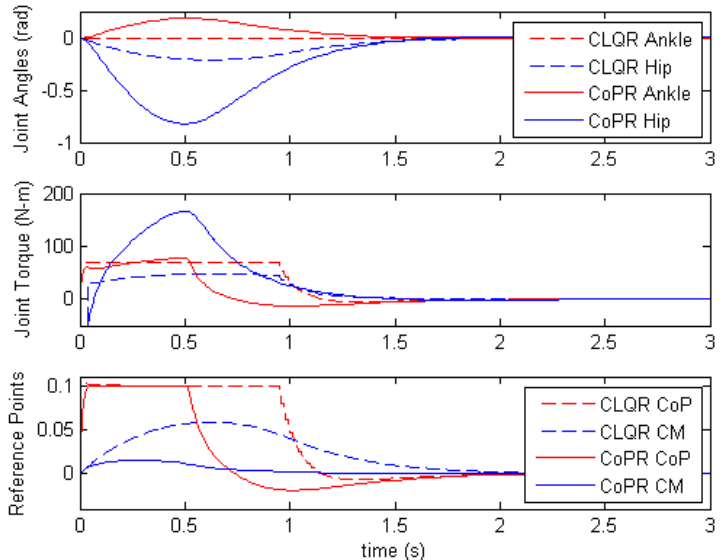

Fig. 9. Comparing the Constrained Linear Quadratic Regulator (CLQR) to the center of pressure regulator (CoPR) for a $17 \mathrm{Ns}$ impulse. The CLQR used a $1.0 \mathrm{~s}$ lookahead while the CoPR used the same control parameters given in Table I

disturbance forces. The constrained LQR controller performs slightly better than the LQR controller with ankle saturation limit because the receding horizon optimization keeps it from exceeding the ankle torque limit. Our controller performs better, able to reject a $23 \mathrm{Ns}$ impulse opposed to the $21 \mathrm{Ns}$ impulse survived by the constrained LQR controller. The response due to our controller is noticeably different because of the use of the regulator on the center of mass, as shown in the animations in Fig. 7 and responses in Fig. 9. The difference can also be seen in Fig. 8, where our controller bends at the hip to recover.

\section{B. Human Balance Experiments}

Here we compare the responses produced by our controller to human balance experiments. The subject was placed with each foot on a force plate to measure ground reaction forces and a motion capture system was used to measure joint movements. A horizontal push was applied from behind; however, the magnitude and duration of the actual push were not measured. We simulate the push as a constant force over
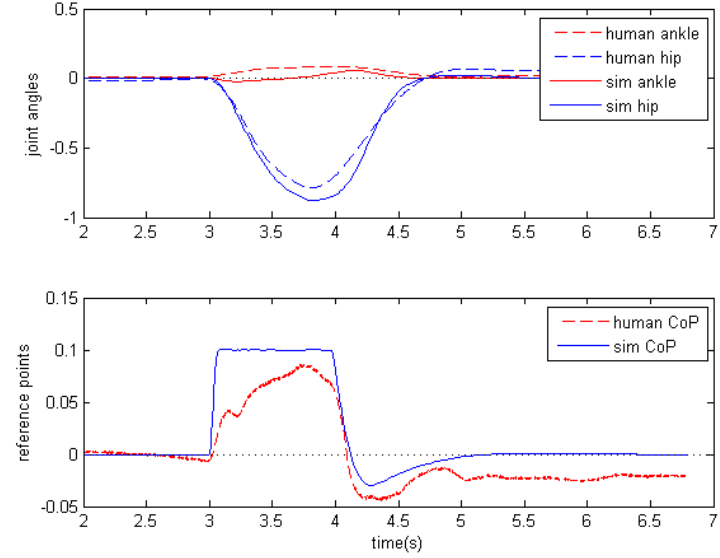

Fig. 10. Comparison of the response of the subject and a simulated double inverted pendulum to a push from behind to the middle of the torso.

TABLE II

CONTROL PARAMETERS FOR HUMAN COMPARISON

\begin{tabular}{|l|r|}
\hline Parameter & Value \\
\hline$m_{1}$ & $25 \mathrm{~kg}$ \\
$m_{2}$ & $45 \mathrm{~kg}$ \\
$L_{1}$ & $0.9 \mathrm{~m}$ \\
$L_{2}$ & $0.5 \mathrm{~m}$ \\
Force & $205 \mathrm{~N} / 0.1 \mathrm{~s}$ \\
$k_{\mathrm{CMp}}$ & 100 \\
$k_{\mathrm{CMd}}$ & 5 \\
$k_{\mathrm{CoP}}$ & 100 \\
$K_{\tau}$ & $\operatorname{diag}\left[1 e^{4}, 1 e^{4}\right]$ \\
\hline
\end{tabular}

a fixed duration rather than an impulse. A double pendulum was fit to the subject by attaching the joints to the center point between the feet, the middle of the hip, and the middle of the shoulders and projecting into the sagittal plane. The plots in Fig. 10 compare the simulated response to the human response using the model parameters and controller gains shown in Table II. The joint angles reflect a response that does not appear to be a simple LQR-like control strategy, but rather a strategy that is closer to that achieved by our 


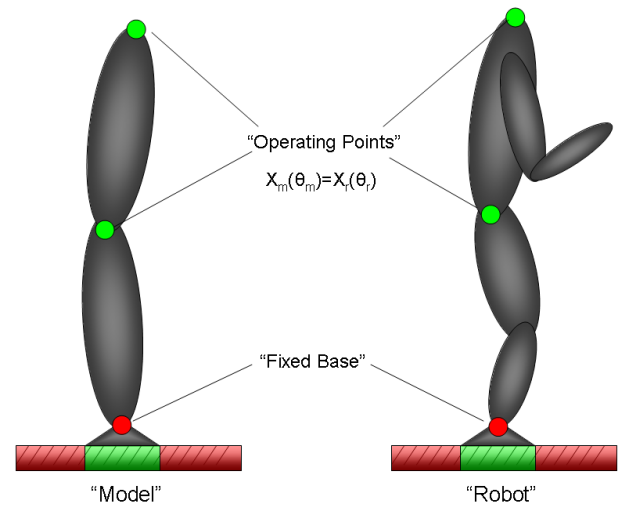

Fig. 11. Operating points are placed on the robot so that it will mimic the simpler model.

controller.

\section{Model Tracking Control}

We would like to control our humanoid robots to behave like a double inverted pendulum. Since a humanoid generally has more degrees of freedom, we can take advantage of its redundancy to accomplish this. The method below shows a general approach to controlling complex robots to track a double inverted pendulum model using our controller to balance.

First, we create operating points to relate the model and the robot, as shown in Fig. 11 where a double pendulum is fit to a triple pendulum that represents a planar biped. The operating points can be written as

$$
\begin{aligned}
P_{m} & =X_{m}\left(\theta_{m}\right) \\
P_{r} & =X_{r}\left(\theta_{r}\right),
\end{aligned}
$$

where $(\cdot)_{m}$ and $(\cdot)_{r}$ are the generalized coordinates of the model (e.g. double pendulum) and the robot (e.g. 3-link planar biped), respectively. These operating points connect the hip of the biped to the middle joint of the double inverted pendulum and the head to the tip. The derivatives of these operating points are

$$
\begin{aligned}
\dot{P}_{m} & =J_{m}\left(\theta_{m}\right) \dot{\theta}_{m} \\
\dot{P}_{r} & =J_{r}\left(\theta_{r}\right) \dot{\theta}_{r},
\end{aligned}
$$

where $J_{m}$ and $J_{r}$ are Jacobians. The objective of the controller is to match these points on the robot to the points on a simulated model. For this we need to relate the motion of these points to the dynamics of the system. The second derivative of the robot operating points can be written as

$$
\begin{aligned}
\ddot{P}_{r} & =\dot{J}_{r} \dot{\theta}_{r}+J_{r} \ddot{\theta}_{r} \\
& =\dot{J}_{r} \dot{\theta}_{r}+J_{r} M_{r}^{-1}\left(\tau_{r}-N_{r}\right),
\end{aligned}
$$

where $M_{r}$ is the inertia matrix of the robot, $N_{r}$ is the gravitational and centrifugal/coriolis forces and $\tau_{r}$ are the joint torques. A feedback controller of the following form is used

$$
\tau_{r}=N_{r}+M_{r} \bar{J}_{r}\left(\ddot{P}_{r}^{d}-\dot{J}_{r} \dot{\theta}_{r}+K_{p} e+K_{d} \dot{e}\right),
$$

where $\bar{J}_{r}$ is the dynamically consistent generalized inverse [16] of $J_{r}, e$ is the error between the operating points of the model and the robot, $e=P_{m}-P_{r}$, and $\ddot{P}_{r}^{d}$ is a desired acceleration. We generally expect $J_{r}$ to have sufficient rank because the robot has more degrees of freedom than the model. This controller reduces to the following differential equation,

$$
\ddot{e}+K_{d} \dot{e}+K_{p} e=0,
$$

which asymptotically drives the error to zero assuming $K_{p}$ and $K_{d}$ are chosen accordingly.

To realize the balance task being performed by the model, which is using the controller from the previous section, we use the acceleration induced by its control,

$$
\ddot{P}_{r}^{d}=\ddot{P}_{m}=\dot{J}_{m} \dot{\theta}_{m}+J_{m} M_{m}^{-1}\left(\tau_{m}-N_{m}\right)
$$

Since the robot may have a center of mass slightly to the side due to its bent legs, we also need to feed in the CM of the robot to a slow integrator that adjusts the desired $\mathrm{CM}$ of the model,

$$
C M_{\text {model }}^{d}=-K_{i} \int_{0}^{t} C M_{\text {robot }} d t
$$

To summarize, the full state of the system is

$$
X=\left(\begin{array}{c}
\theta_{r} \\
\dot{\theta}_{r} \\
\theta_{m} \\
\dot{\theta}_{m} \\
\tau_{m} \\
C M_{\text {model }}^{d}
\end{array}\right)
$$

and the full controller is

$$
\begin{aligned}
\tau_{r}= & N_{r}+M_{r} \bar{J}_{r}\left(\dot{J}_{m} \dot{\theta}_{m}+J_{m} M_{m}^{-1}\left(\tau_{m}-N_{m}\right)\right. \\
& \left.-\dot{J}_{r} \dot{\theta}_{r}+K_{p} e+K_{d} \dot{e}\right),
\end{aligned}
$$

where $\tau_{m}$ is the torque found by simultaneous forward simulation of the model and the integral of Eq.(7).

\section{Simulation Results}

To illustrate the use of this control algorithm, we control the 3-link planar biped using the same double inverted pendulum controller from above. The two systems can be seen in Fig. 12. At the start of the simulation, the double inverted pendulum is fit to the biped using a mapping created by setting the operating points of the two models equal,

$$
\begin{aligned}
\theta_{m} & =X_{m}^{-1}\left(X_{r}\left(\theta_{r}\right)\right) \\
\dot{\theta}_{m} & =\bar{J}_{m} J_{r} \dot{\theta}_{r}
\end{aligned}
$$

The parameters of the robot and model are taken from a biped robot in our lab and are displayed in Table III.

Fitting the model to the robot is an important step. We want the dynamics of the model to match the dynamics of 

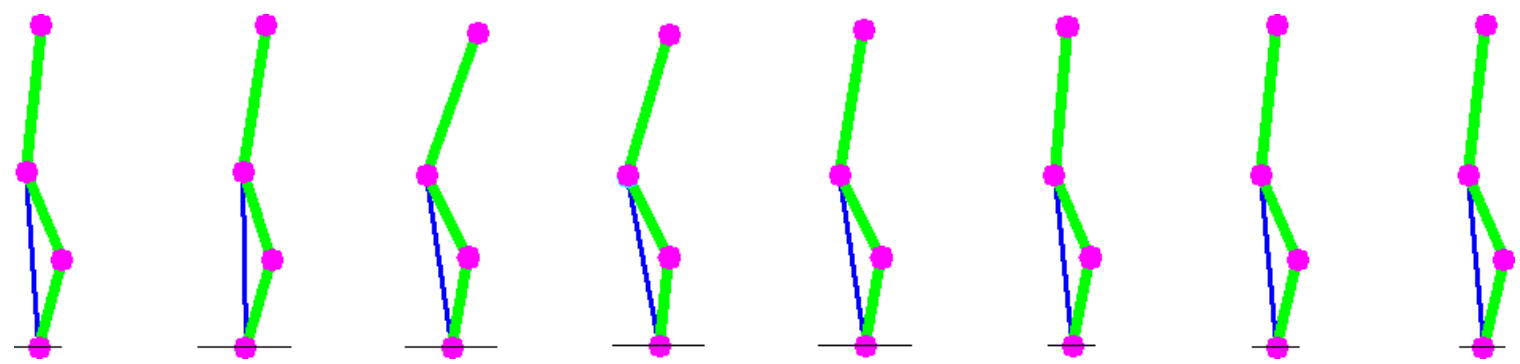

Fig. 15. Animation of the 2-link model and the 3-link robot. The robot is pushed subject to a $100 \mathrm{~N}$ force for $0.1 \mathrm{~s}$. The green dot is the CoP of the 2 -link model and the black dot is the CoP of the robot.

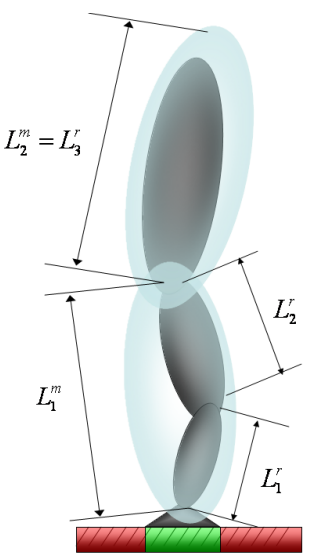

Fig. 12. A double pendulum model fitted to a full planar biped robot
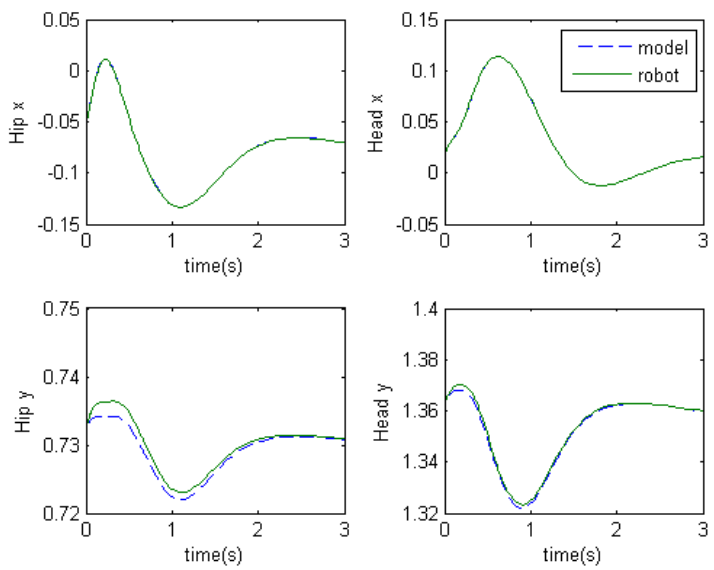

Fig. 13. Cartesian coordinates of the operational space for the model and robot.

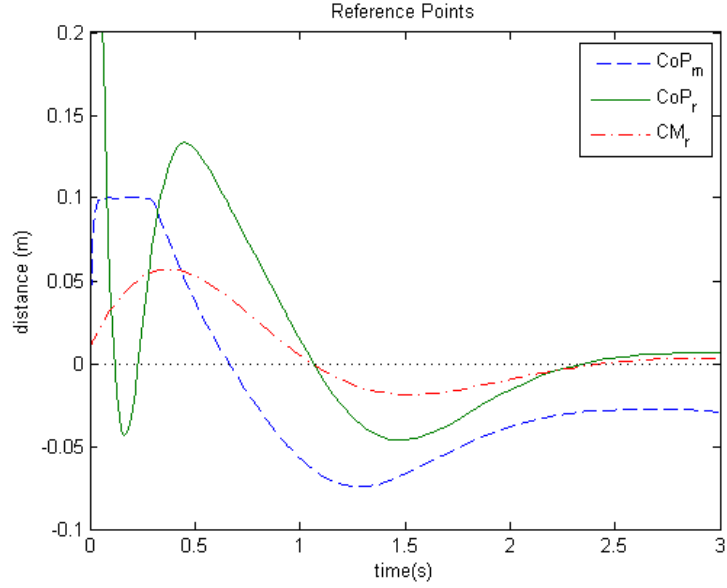

Fig. 14. Reference points response to $17 \mathrm{Ns}$ impulse

the robot as closely as possible. So to do this, the following relationships were used:

$$
\begin{aligned}
L_{1}^{m} & =0.95\left(L_{1}^{r}+L_{2}^{r}\right) \\
L_{2}^{m} & =L_{3}^{r} \\
m_{1}^{m} & =m_{1}^{r}+m_{2}^{r} \\
m_{2}^{m} & =m_{3}^{r}
\end{aligned}
$$

where these variables are described in Fig. 12. The bottom link is purposely set to a slightly shorter length than the sum of the lengths of the femur and tibia to prevent the knees straightening out. Such a condition would be problematic as a singularity exists when the knees are straight and we do not currently have constraints that would keep the knees from bending in the opposite direction. The tracking controller uses PD gains to follow the operational points. The Cartesian positions of these points are shown in Fig. 13. The gains used for this simulation were

$$
\begin{gathered}
K_{p}=\operatorname{diag}[10,10,10,10] \quad K_{d}=\operatorname{diag}[10,10,10,10] \\
K_{i}=2.5
\end{gathered}
$$

While this model tracking controller is able to closely follow the double inverted pendulum model simulation, it does not fully account for the constraints on the CoP. As can be seen in Fig. 14, the CoP of the robot deviates slightly from the $\mathrm{CoP}$ of the model during the transient response and 
could cause instability. However, the correction made by the use of Eq.(14) regulates the center of mass to end up over the ankles and perfectly balance the robot in steady state.

\section{SUMMARY AND FUtURE WORK}

The humanoid balance controller presented here is based on prior work on balancing unstable systems such as inverted pendulums and the acrobot and is inspired by the hip and ankle strategies observed in human balance experiments. The use of a linear quadratic regulator mimics the ankle strategy by causing the robot to behave like a single inverted pendulum while a regulator on the horizontal location of the center of mass resembles a hip strategy controller. The center of pressure serves as a useful ground reference point as it has physical meaning and can be used as a measure of stability. We presented a decoupled controller that tracks the center of pressure and keeps the robot standing at an upright posture. By accounting for the limits on the location of the center of pressure, we ensure that the robot can stand with its feet flat on the ground and withstand large disturbances.

For applying this balancing controller to complex robot systems, we presented a general model tracking control algorithm. For a model system, we used the double inverted pendulum. Using operational points attached to key locations, we derived a feedback controller that allows the robot system to track the model system. The model is simulated and its dynamics are used as a feed-forward term in the control of the robot. An important task in the use of this control algorithm is the choice of the model system that closely approximates the robot. Because of the discrepancies, an additional integrated state was added to adjust the center of mass to end up directly over the ankle, where the moment caused by gravity goes to zero.

All current results are from numerical simulation. The simulation assumed a continuous dynamic system and all analysis was performed in MATLAB. Results were compared to human balance experiments, showing that the subject exhibited a response similar to that generated by our controller. The controller will soon be applied to a full-body hydraulic humanoid robot made by SARCOS. While this paper specifically addresses the problem of balancing, the regulation of the center of pressure and disturbance rejection properties that our controller exhibits are useful in walking and other dynamic motions, which will be the focus of future research.

\section{ACKNOWLEDGMENTS}

The author would like to thank his advisor, Dr. Christopher Atkeson, for his guidance and review comments and the
National Science Foundation (NSF) Integrative Graduate Education and Research Traineeship (IGERT) program for funding (DGE-0333420) this research. This material is also based upon work supported in part by the DARPA Learning Locomotion Program and the National Science Foundation under NSF Grants CNS-0224419, ECS-0325383 and EEC0540865. Balance experiments were performed at the University of Pittsburgh Human Movement and Balance Laboratory.

\section{REFERENCES}

[1] F. Horak and L. Nashner, "Central programming of postural movements: adaptation to altered support-surface configurations." Journal of Neurophysiology, vol. 55, no. 6, pp. 1369-1381, 1986.

[2] M. Vukobratovic, A. A. Frank, and D. Juricic, "On the stability of biped locomotion," IEEE Transactions on Biomedical Engineering, pp. 25-36, January 1970.

[3] C. Galliday and H. Hemami, "Postural stability of the two-degreeof-freedom biped by general linear feedback," IEEE Transactions on Automatic Control, vol. 21, no. 1, pp. 74-79, February 1976.

[4] H. Hemami and B.-R. Chen, "Stability analysis and input design of a two-link planar biped," The International Journal of Robotics Research, vol. 3, no. 2, pp. 93-100, 1984.

[5] A. Goswami, "Postural stability of biped robots and the foot-rotation indication (fri) point," International Journal of Robotics Research, vol. 18, no. 6, pp. 523-533, June 1999.

[6] M. Popovic, A. Goswami, and H. Herr, "Ground reference points in legged locomotion: Definitions, biological trajectories and control implications," International Journal of Robotics Research, vol. 24, no. 12, pp. 1013-1032, December 2005.

[7] K. Hirai, M. Hirose, Y. Haikawa, and T. Takenaka, "The development of honda humanoid robot," in Proceedings of the 1998 IEEE International Conference on Robotics and Automation, vol. 2, May 1998, pp. 1321-1326.

[8] M. Spong, "The swing up control problem for the acrobot," IEEE Control Systems Magazine, vol. 15, no. 1, pp. 49-55, February 1995.

[9] A. Kuo, "An optimal control model for analyzing human postural balance," IEEE Transactions on Biomedical Engineering, vol. 42, no. 1, pp. 87-101, January 1995.

[10] S. Kajita, F. Kanehiro, K. Kaneko, K. Fujiwara, K. Harada, K. Yokoi, and $\mathrm{H}$. Hirukawa, "Biped walking pattern generation by using preview control of zero-moment point," in Proceedings of the IEEE International Concference on Robotics and Automation, vol. 2, September 2003, pp. 1620- 1626.

[11] J. Maciejowski, Predictive Control with Constraints. Prentice Hall, June.

[12] G. Goodwin, M. Seron, and J. D. Dona, Constrained Control and Estimation: An Optimisation Approach. London: Springer-Verlag, 2005.

[13] K. Kondak and G. Hommel, "Control and online computation of stable movement for biped robots," in IEEE/RSJ International Conference on Intelligent Robots and Systems, vol. 1, October 2003, pp. 874-879.

[14] O. Khatib, "A unified approach for motion and force control of robot manipulators: The operational space formulation," IEEE Journal of Robotics and Automation, vol. 3, no. 1, pp. 43-53, February 1987.

[15] J. E. Pratt, "Virtual model control of a bipedal walking robot," Master's thesis, Massachusettes Institute of Technology, 1995.

[16] O. Khatib, L. Sentis, J. Park, and J. Warren, "Whole-body dynamic behaviour and control of human-like robots," International Journal of Humanoid Robotics, vol. 1, no. 1, pp. 29-43, 2004.

[17] L. Sentis and O. Khatib, "A whole-body control framework for humanoids operating in human environments," IEEE Conference on Robotics and Automation, pp. 2641-2648, May 2006. 